\title{
DECELERATING PRESHOCK SEISMIC DEFORMATION IN FAULT REGIONS DURING CRITICAL PERIODS
}

\author{
Papazachos C., Scordilis E., Karakaisis G. and Papazachos B. \\ Department of Geophysics, School of Geology, Aristotle University of Thessaloniki, 54621 , \\ Thessaloniki,costas@lemnos.geo.auth.gr,manolis@geo.auth.gr,karakais@geo.auth.gr
}

\begin{abstract}
We examine the behavior of the intermediate magnitude preshock activity in the fault region of a mainshock during the critical period, that is, in the period when accelerating seismic activity is observed in a broader region (critical region). For this purpose, data concerning 10 recent strong mainshocks ( $M=6.6-8.3$ ) occurred since 1981 in Greece, Anatolia, Japan and California are used. It is observed that during the critical preshock period, decelerating seismic crustal deformation (Benioff strain) is observed in the fault region (within the fault zone and its close vicinity), whereas accelerating deformation is observed in the broader (critical) region. The dimension of the fault region where decelerating deformation is observed scales positively with the mainshock magnitude and negatively with the mean seismicity rate of this region. The duration of this decelerating deformation scales also negatively with the mean seismicity rate. The physical explanation and importance of these results for earthquake prediction are discussed.
\end{abstract}

\section{INTRODUCTION}

Several seismicity patterns have been proposed as precursors of strong earthquakes, but two of them have been much discussed and considered as promising for earthquake prediction. The first of these concerns a premonitory seismic excitation observed in a very broad region around the epicenter of an oncoming mainshock and the second the seismicity quiescence in the epicentral zone. The two patterns together form what has been called by Mogi (1979) as "doughnut pattern". Other patterns of relative locations of zones of excitation and zones of quiescence have been also proposed (e.g. Tzanis and Vallianatos, 2003).

The critical earthquake model has been proposed (Sornette and Sornette, 1990; Sornette and Sammis, 1995) to explain accelerating generation of intermediate magnitude shocks observed before strong mainshocks. According to this model, the physical process of generation of these moderate magnitude preshocks is considered as a critical phenomenon, culminating in a large event (mainshock), which is considered as a critical point. This phenomenon is expressed by the following power-law relation:

$$
S=A+B\left(t-t_{c}\right)^{m}
$$

where $S$ is the cumulative Benioff strain (square root of seismic energy) released in the preshock (critical) region during the preshock period, $t$ is the time to the mainshock, $t_{c}$ is the origin time of the mainshock and $A, B, m$ are parameters which are determined by the available data for preshocks (Bufe and Varnes, 1993).

In the present work we examine the time variation of the seismic deformation in the fault (rupture) zone and its close vicinity (fault region) during the critical (preshock) period when accelerating seismic deformation occurs in the broader (critical) region.

\section{BACKGROUND}

Bowman et al. (1998) quantified the degree of deviation from linearity and of the accelerating with the time to the mainshock Benioff strain by defining a curvature parameter, $\mathrm{C}$, as the ratio of the root mean square error of the power-low fit (relation 1) to the corresponding linear fit error. $\mathrm{Pa}$ pazachos and Papazachos $(2000,2001)$ suggested the use of elliptical critical regions and several 
constraints to the critical earthquake model expressed by four empirical relations. Recently, $\mathrm{Pa}$ pazachos et al. (2004a) extended this work by using data from areas of a variety of seismotectonic regions (Aegean, Anatolia, Himalayas, Japan, California) and proposed the following four empirical relations:

$$
\begin{array}{cc}
\log R=0.42 M-0.30 \log s_{r}+1.28, & \sigma=0.11 \\
\log t_{p}=3.87-0.45 \log s_{r}, & \sigma=0.10 \\
\log \left(\frac{A}{t_{p}}\right)=1.01 \log S_{r}, & \sigma=0.04 \\
M=M_{13}+0.6, & \sigma=0.15
\end{array}
$$

where $M$ is the magnitude of the mainshock, $R$ (in $\mathrm{km}$ ) is the radius of the circle with area equal to the area of the elliptical critical region, $t_{p}$ (in years) is the duration of the preshock sequence, $S_{r}$ (in Joule $e^{1 / 2} / \mathrm{yr}$ ) is the long term rate of Benioff strain in the critical region, $\mathrm{s}_{\mathrm{r}}$ is the same rate per $10^{4} \mathrm{~km}^{2}$ (seismicity rate), $A$ is the parameter of relation (1), $M_{13}$ is the average magnitude of the three largest preshocks and $\sigma$ is the corresponding standard deviation. Relations (2), (3), (4) and (5) can be used to predict the magnitude of an ensuing mainshock because $R, \log s_{r}$ and $M_{13}$ are known before its generation. The minimum magnitude, $M_{\min }$, for which the best solution is obtained, is given by the relation:

$$
M-M_{\min }=0.54 M-1.91, \quad \sigma=0.08
$$

The degree of agreement of the calculated parameters $R, t_{p}, M, A$ for an elliptical (or circular) region under examination, with the corresponding values of these parameters given by relations $(2$, $3,4,5$ ), which hold for known preshock regions, is quantified by a probability $P$. This parameter has been defined as the mean value of the probabilities that each of these four parameters attains a value close to its expected one by these relations, assuming that the deviation of each parameter follows a Gaussian distribution (Papazachos and Papazachos, 2001). In order to quantify the degree of agreement of the calculated four parameters of an examined region with all previously mentioned characteristics of the critical earthquake model, a quality index, $q$, has been proposed (Papazachos et al., 2002) which is given by the relation:

$$
q=\frac{P}{m C}
$$

This parameter is of importance for defining the center, $Q$, of the critical region. Thus, by calculating the $q$ value in a grid of geographic points with the desired density (e.g. $0.2^{0} \mathrm{NS}, 0.2^{0} \mathrm{EW}$ ), we define as center of the critical region the point for which $q$ takes its maximum value, since this value simultaneously evaluates: a) the compatibility of the examined time variation of Benioff strain with the behavior of such variations observed before past mainshocks (large P), b) the degree of acceleration of this strain (small $C$ and small $m$ ). A representative observed value of $m$ is 0.30 , in agreement with theoretical results, while valid solutions are considered those for which $\mathrm{C} \leq 0.60$ and $\mathrm{q} \geq 3.0$.

Valid accelerating seismic deformation $(C<0.60, m=0.30)$ cannot be identified until a certain time, $t_{i}$, which is called "identification time" and represents the earliest time up to when the available data for a preshock sequence give a valid solution (Papazachos et al., 2004a). This time, $t_{i}$, which is known before the generation of the mainshock, is given by the relation:

$$
t_{c}=t_{i}+\exp \left(7.09-1.04 \log s_{r}\right), \quad \sigma=1.7 y r s
$$

This formula can be used for estimating the origin time, $t_{c}$, of an ensuing mainshock. It is possible to obtain better results using a different constant for equation (8) calculated for each area, since the reported value $(=7.09)$ is a globally representative average.

The geographic distribution of the $q$ values can give important information on the location of the epicenter, $E$, of the oncoming mainshock (Papazachos et al., 2004b). Thus, the distance, $x$ (in km), between the epicenter of the ensuing mainshock and of the geographical point, $Q$, for which the quality parameter, q, has its largest value and which is considered as the center of the critical region is given by the relation: 


$$
x=1150-160 \log s_{r}, \quad \sigma=60 \mathrm{~km}
$$

It has been further observed that during the preshock period $Q$ changes positions but relation (9) is still applicable. This is due to the fact that seismic quiescence in the fault region forces the center, $Q$, of the seismic excitation to move away from the real epicenter at a distance which remains almost constant during the whole critical period and is given by this relation. Preliminary application of this technique to already occurred mainshocks in Aegean, Anatolia, Japan and California indicates an uncertainty of $150 \mathrm{~km}$ with high ( $95 \%)$ typical confidence.

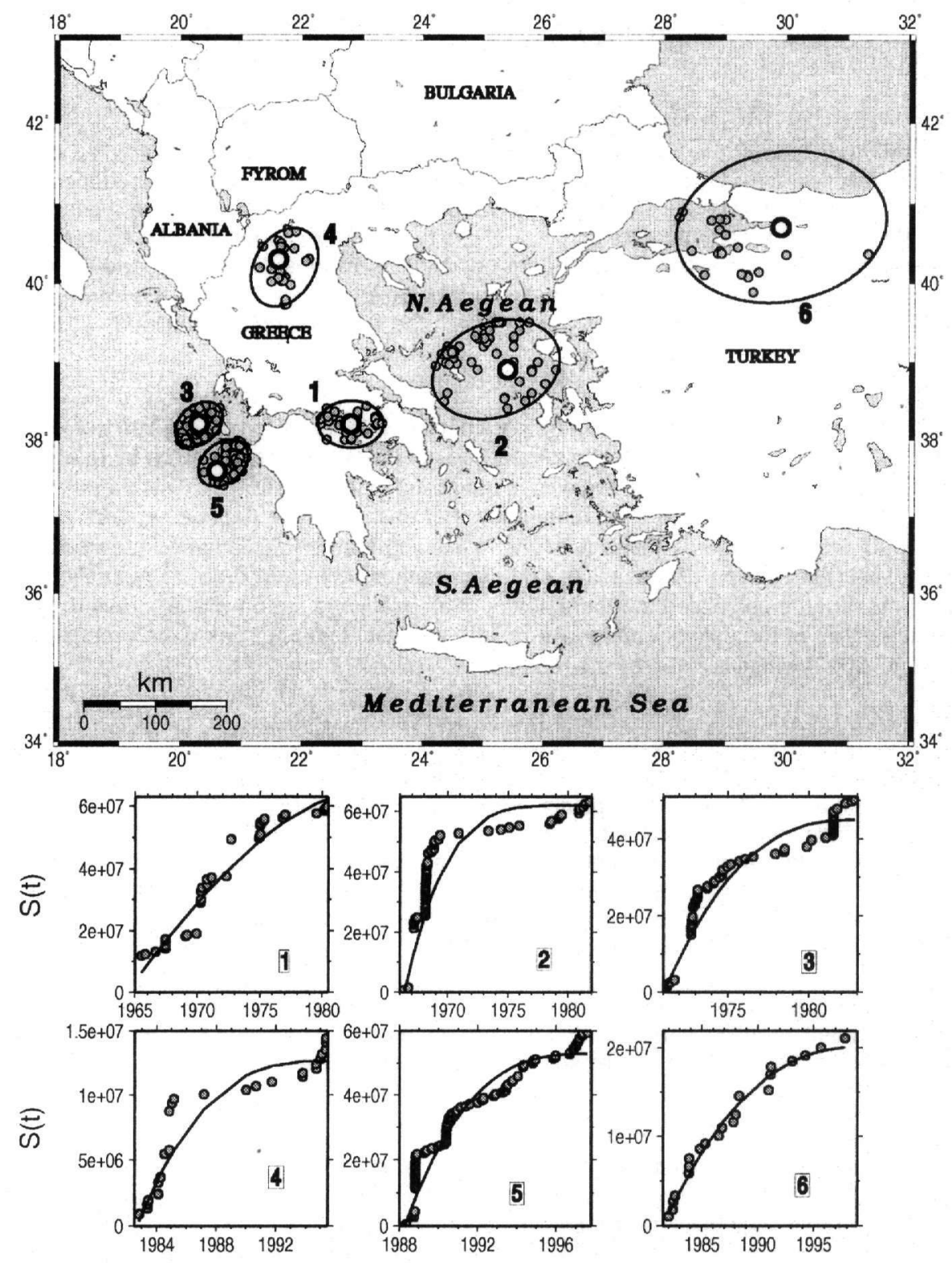

Figure 1.- Elliptical fault regions for five mainshocks in Greece $(1,2,3,4,5)$ and one mainshock in Anatolia (6). The epicenters of the mainshock are denoted by large circles, while the smaller circles show the epicenters of preshocks (upper part). Decelerating time variation of the cumulative Benioff strain, $\mathrm{S}$ (in Joule ${ }^{1 / 2}$ ), for each area released by preshocks (lower part). 


\section{DATA AND PROCEDURE}

To test the behavior of the seismic crustal deformation (Benioff strain) in the fault region during the preshock (critical) period proper reliable data must be used. These data must be global, complete, homogeneous and accurate as possible. These properties concern the mainshocks as well as preshocks and are better satisfied if data of recent earthquakes are used. Thus, the sample of mainshocks is formed of strong earthquakes $(M>6.5)$, which occurred during the last fifteen years.

Table 1. Information on the area, date, epicenter coordinates, magnitude of the 10 mainshocks and on the parameters of the preshock decelerating seismic deformation in the fault region of these mainshocks. sr (in Joule $1 / 2 / \mathrm{yr} .104 \mathrm{~km} 2$ ) is the long term deformation rate, a (in $\mathrm{km}$ ) is the length of the large axis of the elliptical fault region and $z$ is the azimuth of this axis. $C$ is the curvature of the time variation of the Benioff strain, $m$ is the parameter of relation (1) and qd is the quality index defined by relation (13). Mmin is the minimum preshock magnitude, $\mathrm{n}$ is the number of preshocks and ts is the start year of the preshock sequence.

\begin{tabular}{|c|c|c|c|c|c|c|c|c|c|c|c|c|}
\hline Area & Date & $\varphi, \lambda$ & $M$ & $\log _{\mathrm{r}}$ & $a$ & $z$ & C & $\mathrm{m}$ & $q_{d}$ & $\overline{M_{\min }}$ & $n$ & $t_{s}$ \\
\hline \multicolumn{13}{|c|}{ Greece } \\
\hline 1 & 198102,24 & $38.2,22.8$ & 6.7 & 6.00 & 47 & 90 & 0.45 & 2.7 & 3.3 & 4.3 & 33 & 1965 \\
\hline 2 & 198112,19 & $38.9,25.2$ & 7.2 & 6.00 & 94 & 70 & 0.57 & 3.2 & 3.8 & 4.5 & 58 & 1966 \\
\hline 3 & 198301,17 & $38.2,20.3$ & 7.0 & 6.20 & 38 & 50 & 0.42 & 2.8 & 1.7 & 4.5 & 54 & 1971 \\
\hline 4 & 199505,13 & $40.2,21.7$ & 6.6 & 6.10 & 59 & 30 & 0.54 & 3.2 & 2.7 & 4.2 & 21 & 1981 \\
\hline 5 & 199711,18 & $37.7,20.7$ & 6.6 & 6.30 & 40 & 50 & 0.50 & 3.0 & 3.0 & 4.2 & 98 & 1988 \\
\hline \multicolumn{13}{|c|}{ Anatolia } \\
\hline $\begin{array}{l}6 \\
\text { Japa }\end{array}$ & 199908,17 & $40.7,29.9$ & 7.4 & 5.70 & 146 & 80 & 0.42 & 3.2 & 5.3 & 4.6 & 21 & 1979 \\
\hline 7 & 199410,04 & $43.8,147.6$ & 8.3 & 6.40 & 162 & 50 & 0.44 & 3.3 & 7.3 & 5.2 & 51 & 1984 \\
\hline \multicolumn{13}{|c|}{ California } \\
\hline 8 & 198910,18 & $36.9,-122.0$ & 7.0 & 5.50 & 58 & 170 & 0.34 & 3.4 & 4.3 & 4.2 & 27 & 1964 \\
\hline 9 & 199204,25 & $40.2,-124.1$ & 7.2 & 5.50 & 96 & 170 & 0.57 & 2.5 & 2.5 & 4.5 & 67 & 1967 \\
\hline 10 & 199206,28 & $34.2,-116.5$ & 7.3 & 5.50 & 148 & 140 & 0.59 & 3.3 & 3.5 & 4.6 & 24 & 1957 \\
\hline
\end{tabular}

This data set includes the five largest mainshocks ( $M \geq 6.5)$ in Greece since 1981, the three largest earthquakes $(M \geq 7.0)$ in California since 1989, the largest earthquake $(M=8.3)$ in Japan since 1990 and the largest earthquake (M=7.4) in Anatolia since 1990. The date, geographic coordinates of epicenter and moment magnitude for these ten shallow mainshocks are listed in table (1).

Since in all these areas, durations of preshock (critical) periods are less than three decades (11yrs for Japan, 13yrs for Greece, 17yrs for Anatolia and 27yrs for California, Papazachos et al., 2004a), all preshocks for which data are used in the present paper occurred after the installation (1965) of reliable international networks. Therefore, epicenter coordinates and magnitudes for these preshocks are accurate in a satisfactory degree for the purpose of the present work. The magnitudes are given in several scales $\left(M_{L}, m_{b}, M_{S}, M w\right)$. Proper formulae have been used (Papazachos et al., 1997; Scordilis, 2004) to transform all available values of magnitudes to moment magnitude, $\mathrm{M}_{\mathrm{W}}$, so that the data used are homogeneous in respect to magnitude. Completeness of the data has been tested through frequency-magnitude plots and plots of time variation of frequency of shocks for several magnitude levels. In this way it has been shown that the data for all four areas are complete for $M \geq 4.0$ since 1970.

An algorithm has been developed to test by these data the validity of the power-law relation (1) and estimate its parameters $(A, B, m)$. The region for which this test has been made in each case has an elliptical shape centered at the epicenter (or very close to the epicenter) of the mainshock. The ellipticity has been kept constant $(e=0.7)$ because several tests have shown that such a shape is the most proper one for identification of seismicity quiescence (Papazachos et al., 2004b), while the azimuth, $z_{1}$ and the length, a (in $\mathrm{km}$ ), of the large axis of the ellipse, as well as the duration, $t_{d}$ (in years), of the preshock sequence have been free to be estimated by a minimization procedure. The curvature parameter, $C$, is calculated for several values of the relative parameters $\left(A, B, a, t_{d}\right.$, etc.) and the group of values of these parameters which corresponds to the smallest $C$ value is considered as best solution. 


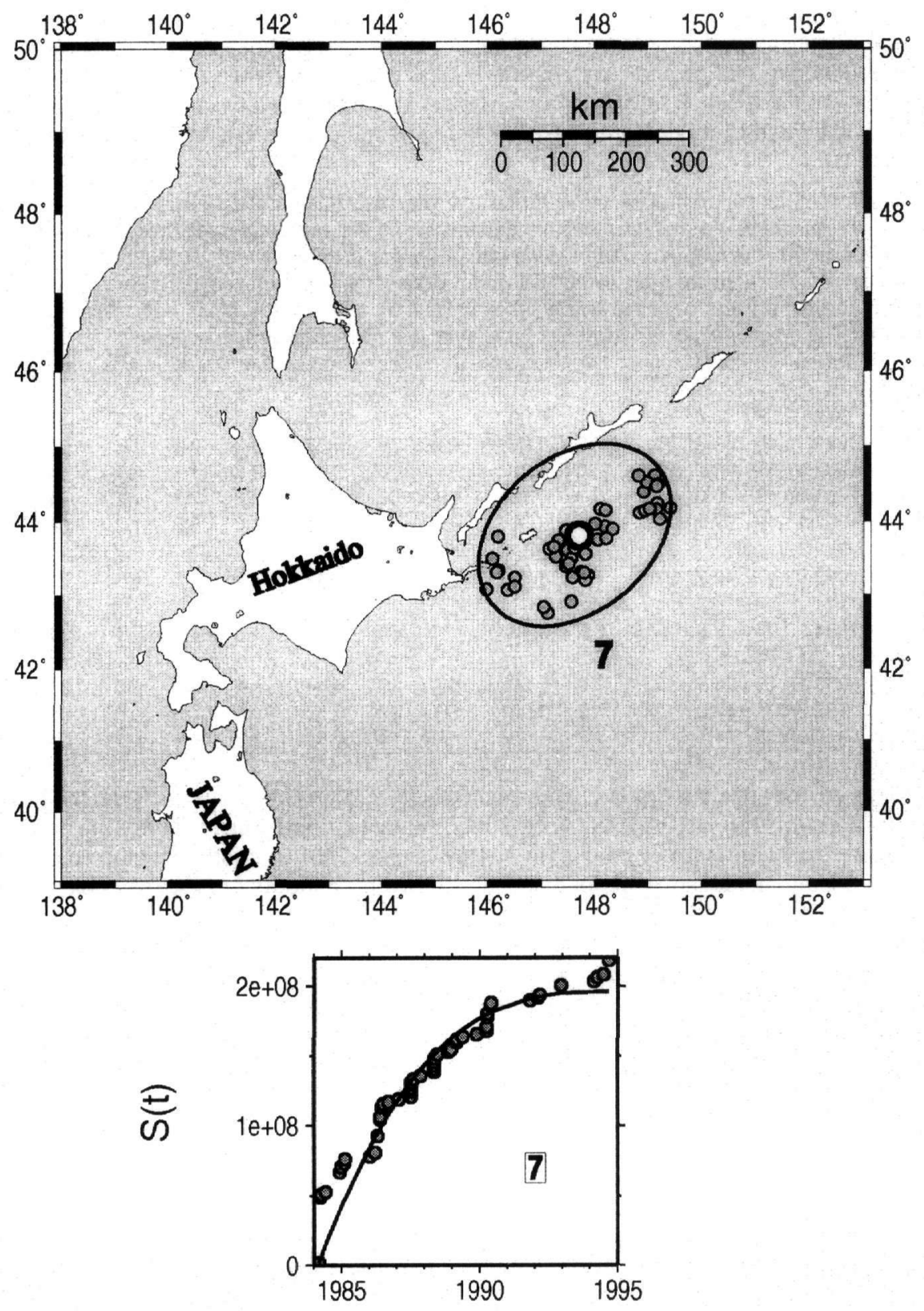

Figure 2.- Elliptical fault region for one mainshock in Japan (upper part) and corresponding time variation of the cumulative Benioff strain released by its preshocks (lower part). Symbols are as in figure (1).

\section{RESULTS}

Table (1) gives information on the best solution (smallest $\mathrm{C}$ value) for each one of the ten preshock sequences. These $\mathrm{C}$ values and the corresponding $\mathrm{m}$ values estimated by the mentioned 
above procedure are listed in this table. It is seen that all $\mathrm{C}$ values are smaller than unit $(\mathrm{C} \leq 0.59$, $\bar{C}=0.48 \pm 0.08)$ and all $m$ values are larger than unit $(m>2.5, \bar{m}=3.1 \pm 0.3)$, which show a clear deceleration of the preshock seismic crustal deformation (Benioff strain) and a good fit of the time variation of this deformation to the power-law expressed by relation (1).

The minimum preshock magnitude, $M_{\min }$, for which a best solution is obtained for each case, is also given on table (1). From these values and from the corresponding values of the mainshock magnitude, $\mathrm{M}$, it comes out that:

$$
M-M_{\min }=0.41 M-0.22, \quad 6.5 \leq M \leq 8.3
$$

in the least squares' sense. This relation shows that decelerating preshock Benioff strain in the fault region with time of the mainshock is due to the decelerating generation of intermediate magnitude preshocks. Comparison of this relation with the relation (6) shows that the cut-off minimum magnitude is clearly larger for the accelerating seismic deformation in the broad critical region than for the decelerating seismic deformation in the narrow fault region. Thus, for mainshocks with magnitudes 7.0 and 8.0 the minimum preshock magnitudes are 5.1 and 5.6 for accelerating deformation and 4.4 and 5.0 for decelerating deformation, respectively. Although this is so, decelerating seismic deformation in the epicentral zone also holds for intermediate magnitude preshocks.

The length of the large axis, a (in $\mathrm{km}$ ), of the elliptical regions for which parameters are listed in table (1) increases with increasing $M$ and decreasing $s_{r}$ similar to the radius, $R$, of the broader (critical) region (relation 2). For this reason and because the available data in the present work (10 values of a) are not enough for an independent reliable estimation of the two scaling coefficients, we adopted the values for these scaling coefficients which hold for the broader critical region and have been also derived theoretically. Thus, by considering these coefficients $(0.42$ for $M$ and -0.30 for $\operatorname{logs}_{r}$ ) and taking into consideration the values of $a$ (in $\mathrm{km}$ ), $M$ and $s_{r}$ listed on table (1) we obtain the relation:

$$
\log a=0.42 M-0.30 \log s_{r}+0.65, \quad \sigma=0.13
$$

From this and relation (2) we find that the linear dimension of the broader (critical) region, where accelerating deformation occurs, is about five times larger than the narrow fault region where decelerating seismic deformation occurs. On the other hand, the length of fault region where decelerating deformation occurs (the double of the elliptical axis a) is on the average two times larger than the length, $L$, of the fault of the mainshock.

From the values of the duration, $t_{d}$, of the decelerating seismic deformation in the fault region and $s_{r}$ listed in table (1) we get:

$$
\log t_{d}=3.90-0.45 \log s_{r}, \quad \sigma=0.05
$$

in the least squares' sense. It shows that decelerating deformation in the fault zone decreases with increasing seismicity. This relation is almost identical with relation (3), which indicates that decelerating deformation in the fault region occurs in the same period when accelerating deformation occurs in the broader (critical) region.

A quality factor, $q_{d}$, can be also defined for the fault region by the relation:

$$
q_{d}=\frac{P m}{C}
$$

where $P$ is the probability that observations are compatible with relations $(11,12)$. The calculated values of $C, m$ and $q_{d}$ for these ten cases investigated in the present paper are listed in table (1). From these values it comes out that:

$$
C<0.60, \quad 2.5 \leq m<3.5, \quad q_{d} \geq 1.7
$$

These inequalities can be used as constraints for considering an investigated fault as potentially candidate for generating a future strong shock.

An identification time, $T_{i}$, can be also defined for the decelerating deformation period. The data presented in this work suggest a relation of the form:

$$
\log t_{c}=\tau_{i}+\exp \left(a_{d}-1.04 \log s_{d}\right), \quad \sigma=1.6 y r s
$$

which can be also used to estimate the origin time, $t_{c}$, of the mainshock. $A$ representative value of $a_{d}$ is 8.18 but it varies between different areas.

The upper part of figure (1) shows the five elliptical regions in Greece (code numbers 1, 2, 3, 4, 5 in table 1) and of the one in western Anatolia (code number 6 ) where decelerating seismic defor- 
mation occurred before the generation of the corresponding mainshocks. The large circle in each zone is the epicenter of the mainshock and smaller circles are the epicenters of the intermediate magnitude preshocks, which occurred in a decelerating mode up to the generation of the corresponding mainshock. In the lower part of this figure, the time variation of the cumulative Benioff strain released by these preshocks is shown. Figure (2) shows such results for preshocks of the mainshock that occurred in Japan (code number 7 in table 1) and figure (3) for the three mainshocks in California (code numbers $8,9,10$ in table 1). Decelerating strain with the time to the mainshock is obvious in all ten cases. Also, fitting of the power-law relation (1) to the data (continuous curve in lower parts of figures $1,2,3$ ) is satisfactory. However, it should be noted that in almost all ten studied cases the decelerated seismicity pattern is followed by an increase of seismicity near the end of the deceleration period (see fig $1,2,3$ ). This increase is probably due to the "classical" foreshocks, which precede each mainshock.

The time variation of the seismic deformation (Benioff strain) is due to the time variation of the frequency of intermediate magnitude preshocks or to the variation of the magnitude of these shocks or to both. To examine this problem we separated each of the critical periods of the ten seismic sequences into ten equal intervals and for each interval we calculated the mean frequency, $n$, of preshocks and the mean maximum magnitude, $M$, of all preshock sequences which occurred in the corresponding broader (critical) region. We repeated the calculations for preshocks that occurred in the narrow fault region. These observations show that the time variation of the frequency of preshocks occurs with a positive rate $(=0.76)$ in the critical region and with a negative rate $(=-$ 0.75 ) in the fault region and that the time variation of the maximum preshock magnitude also occurs with a positive rate $(=0.07)$ in the critical region and with a negative rate $(=-0.06)$ in the fault region. These results lead to the important conclusion that preshock accelerating deformation is due to the increase of both the frequency, as well as the magnitude of preshocks in the critical region and that preshock decelerating deformation is due to the decrease of both the frequency and magnitude of preshocks in the epicentral zone.

\section{CONCLUSIONS AND DISCUSSION}

During the critical (preshock) period, $t_{p}$, when accelerating seismic deformation (Benioff strain) occurs in a broad region (critical region), decelerating seismic deformation occurs in a fault region which includes the fault (rupture) zone of the mainshock and its close vicinity. The critical period decreases with increasing long-term deformation, $s_{r}$ (see relations 3,12 ). This critical period is of the order of one up to a few decades. The dimension of the fault region, where preshock decelerating deformation occurs is about two times larger than the length that the fault zone of the oncoming mainshock and about five times smaller than the critical region (see relations 2,11 ).

Time variations of seismic deformation in the critical region and in the fault region deviate strongly from linearity $(C<0.60)$. Preshock seismic deformation is intensely decelerating in the fault region $(m>2.5)$ and is intensely accelerating in the critical region $(m<0.35)$. Preshock accelerating seismic deformation is due to the increase of the frequency of generation and of magnitude of the preshocks in the critical region, while preshock decelerating deformation is due to decrease of the frequency of generation and of magnitude of preshocks in the fault region.

Attempts for physical interpretation of both patterns of seismic deformation have been proposed. The model of critical earthquake is widely now adopted for rationalizing accelerating preshock deformation in the broad (critical) region (Vanneste and Sornette, 1992; Jaume and Sykes, 1999; Rundle et al., 2000). Seismicity quiescence in the fault zone has been attributed to stress relaxation due to preseismic sliding (Wyss at al., 1981; Dmowska et al., 1988). Such relaxation extends in an area larger than the rupture zone of the oncoming mainshock (Kato et al., 1997), which explains the observational result of the present paper that decelerating seismic deformation occurs in a region with length about two times larger than the fault length. Present work indicates that the two physical processes, whatever they are, take place almost simultaneously in two spatially separated regions and create in both regions observable precursory phenomena.

It is, therefore, evident that a combined use of such observations can improve the relative methods for intermediate term earthquake prediction. Thus, estimations of the magnitude of an oncoming mainshock by relations (2-5) and of its origin time by the relation (8), which result from ac- 
celerating deformation pattern, can be tested by relations (11) and (12), respectively, which result from the deceleration deformation pattern. Also, estimation of the location of a mainshock epicenter on the basis of relation (9) can be improved by using the constraint that fault region has to be in a decelerating deformation state during the critical period. An attempt for a retrospective prediction of the ten mainshocks listed on table (1) by this combined procedure indicates that uncertainties are: \pm 0.4 for the magnitude, \pm 3.5 years for the origin time and less than $150 \mathrm{~km}$ for the epicenter. However, application of this method for predicting future mainshocks, currently under way, is a more objective way of testing the method and estimating its uncertainties.

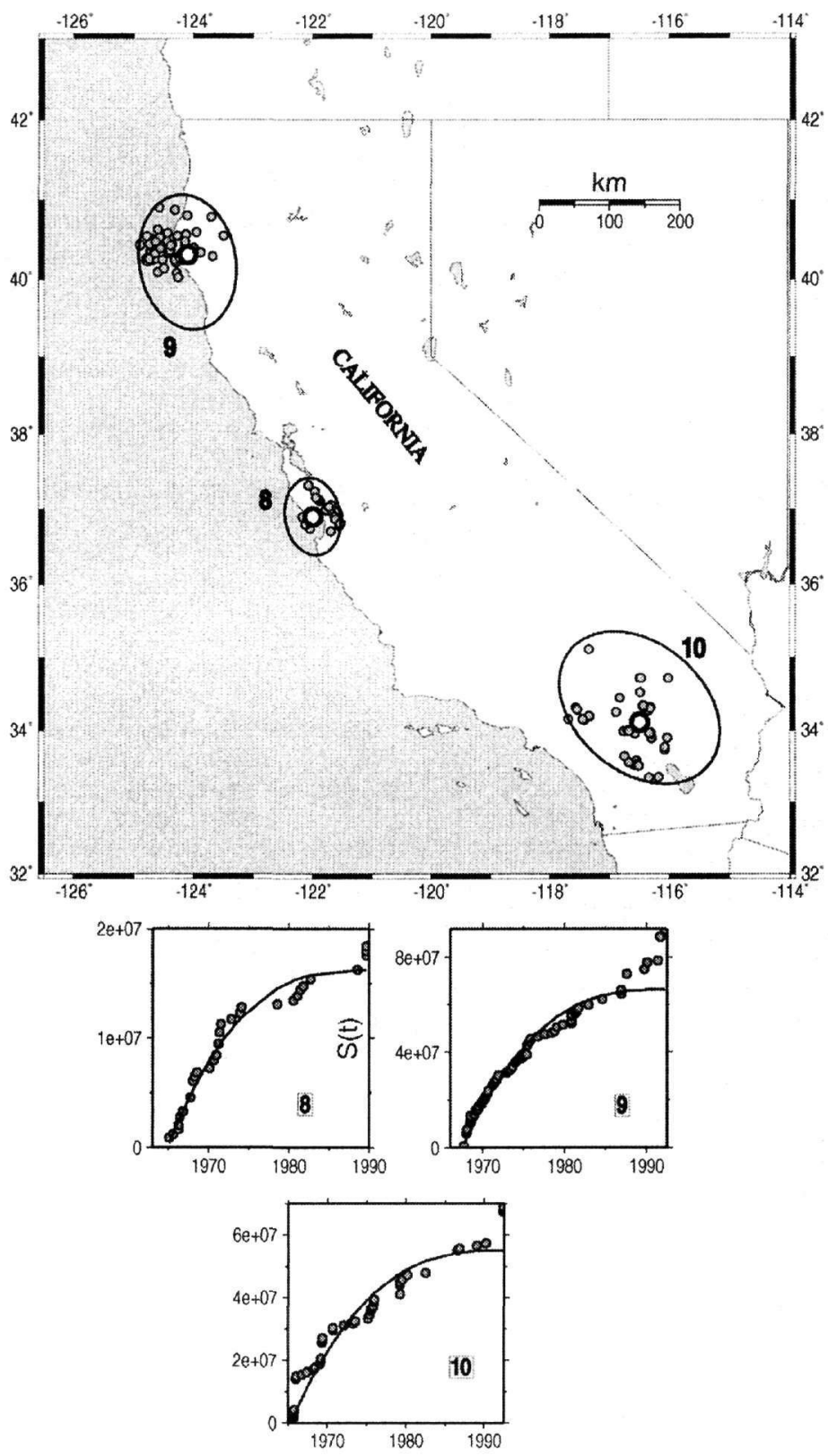

Figure 3.- Elliptical fault regions for three mainshocks in California (upper part) and corresponding time variation of the cumulative Benioff strain released by their preshocks. Symbols are as in figure (1). 


\section{ACKNOWLEDGEMENTS}

The GMT software (Wessel and Smith, 1995) was used to generate the maps of this study. This work has been partially supported by the Greek Planning and Protection Organization (OASP), (Res. Comm. AUTH project 20242) and is a Geophysical Laboratory contribution number \#0638/2004

\section{REFERENCES}

Bowman, D.D., Ouillon,G., Sammis,C.G., Sornette,A. \& Sornette,D. 1998. An observational test of the critical earthquake concept. J. Geophys.Res., 103, 24359-24372.

Bufe, C.G. \& Varnes, D.J. 1993. Predictive modeling of seismic cycle of the Great San Francisco Bay Region. J. Geophys. Res., 98, 9871-9883.

Dmowska, R., Rice, J.R., Lovison, L.C. \& Joselle, D. 1988. Stress transfer and seismic phenomena in coupled subduction zones during the earthquake cycle. J. Geophys. Res., 93, 7869-7884.

Jaume, S.C. \& Sykes, L.R. 1999. Evolving towards a critical point: A review of accelerating seismic moment/energy release rate prior to large and great earthquakes. Pure Appl. Geophys., 155, 279-306.

Kato, N., Ohtake, M \& Hirasawa, T. 1997. Possible mechanism of precursory seismic quiescence: regional stress relaxation due to preseismic sliding. Pure Appl. Geophys., 150, 249-267.

Mogi, K. 1979. Two kinds of seismic gaps. Pure Appl. Geophys., 117, 1172-1186.

Papazachos, B.C., Kiratzi, A.A. \& Karakostas, V.G. 1997. Toward a homogeneous moment magnitude determination in Greece and surrounding area. Bull. Seism. Soc. Am., 87, 474-483.

Papazachos, B.C. \& Papazachos, C.B. 2000. Accelerated preshock deformation of broad regions in the Aegean area. Pure Appl. Geophys., 157, 1663-1681.

Papazachos, B.C., Scordilis, E.M., Papazachos, C.B. \& Karakaisis, G.F., 2004b. Currently active critical regions in parts of the Continental fracture system, J. Seismology, (submitted).

Papazachos, C.B. \& Papazachos, B.C. 2001. Precursory accelerating Benioff in the Aegean area. Ann. di Geofisica, 44, 461-474.

Papazachos, C.B., Karakaisis, G.F., Savvaidis, A.S. \& Papazachos, B.C. 2002. Accelerating seismic crustal deformation in the southern Aegean area. Bul. Seism. Soc. Am., 92, 570-580.

Papazachos, C.B., Karakaisis, G.F., Scordilis, E.M. \& Papazachos, B.C. 2004a. Global observational properties of the critical earthquake model, Bull. Seism. Soc. Am., (submitted).

Rundle, J.B., Klein, W., Turcotte, D.L. \& Malamud, B.D. 2000. Precursory seismic activation and critical-point phenomena. Pure Appl. Geophys., 157, 2165-2182.

Scordilis, E.M. 2004. Magnitude scales revisited: New global relations between $M_{S}, m_{b}, M_{L}$ and moment magnitude. Bull. Seism. Soc. Am. (submitted).

Sornette, A. \& Sornette D. 1990. Earthquake rupture as a critical point. Consequences for telluric precursors. Tectonophysics, 179, 327-334.

Sornette, D. \& Sammis, C.G. 1995. Complex critical exponents from renormalization group theory of earthquakes: implications for earthquake predictions. J.Phys. I. France., 5, 607-619.

Tzanis, A. \& Valianatos, F. 2003. Distributed power law seismicity changes and crustal deformation in the SW Hellenic Arc. Nat. Haz. Earth Sys. Sci., 3, 179-195.

Vanneste, C. \& Sornette, D. 1992. Dynamics of rupture in thermal fuse models. J. Phys. I. Fr., 2, 1621-1644.

Wessel, P. \& Smith, W. 1995. New version of the Generic Mapping Tools. EOS Trans. Amer. Geophys. U., 76:329.

Wyss, M., Klein, F.M.W. \& Johnston, A.C. 1981. Precursory to the Kalapana M=7.2 earthquake. J. Geophys. Res., 86, 3881-3900. 